\title{
A pharmaco-EEG study on antipsychotic drugs in healthy volunteers
}

\author{
Masafumi Yoshimura • Thomas Koenig • \\ Satoshi Irisawa - Toshiaki Isotani $\cdot$ Keizo Yamada • \\ Mitsuru Kikuchi • Gaku Okugawa • Takami Yagyu • \\ Toshihiko Kinoshita • Werner Strik • Thomas Dierks
}

Received: 27 September 2006 / Accepted: 5 February 2007 / Published online: 27 February 2007

(C) Springer-Verlag 2007

\begin{abstract}
Rationale Both psychotropic drugs and mental disorders have typical signatures in quantitative electroencephalography (EEG). Previous studies found that some psychotropic drugs had EEG effects opposite to the EEG effects of the mental disorders treated with these drugs (key-lock principle).

Objectives We performed a placebo-controlled pharmacoEEG study on two conventional antipsychotics (chlorpromazine and haloperidol) and four atypical antipsychotics (olanzapine, perospirone, quetiapine, and risperidone) in healthy volunteers. We investigated differences between conventional and atypical drug effects and whether the drug effects were compatible with the key-lock principle.
\end{abstract}

M. Yoshimura $(\bowtie) \cdot$ T. Koenig $\cdot$ M. Kikuchi $\cdot$ W. Strik $\cdot$ T. Dierks Department of Psychiatric Neurophysiology,

University Hospital of Psychiatry,

Bern, Switzerland

e-mail: yoshimum@takii.kmu.ac.jp

M. Yoshimura $\cdot$ S. Irisawa $\cdot$ T. Isotani $\cdot$ K. Yamada

G. Okugawa $\cdot$ T. Yagyu $\cdot$ T. Kinoshita

Department of Neuropsychiatry, Kansai Medical University,

10-15, Fumizono-cho, Moriguchi,

Osaka 570-8506, Japan

T. Isotani

Department of Psychiatry, Kansai Memorial Hospital,

Osaka, Japan

M. Kikuchi

Department of Psychiatry and Neurobiology,

Graduate School of Medical Science, Kanazawa University,

Kanazawa, Japan

T. Yagyu

Department of Psychiatry, Neyagawa Sanatorium,

Osaka, Japan
Methods Fourteen subjects underwent seven EEG recording sessions, one for each drug (dosage equivalent of $1 \mathrm{mg}$ haloperidol). In a time-domain analysis, we quantified the EEG by identifying clusters of transiently stable EEG topographies (microstates). Frequency-domain analysis used absolute power across electrodes and the location of the center of gravity (centroid) of the spatial distribution of power in different frequency bands.

Results Perospirone increased duration of a microstate class typically shortened in schizophrenics. Haloperidol increased mean microstate duration of all classes, increased alpha 1 and beta 1 power, and tended to shift the beta 1 centroid posterior. Quetiapine decreased alpha 1 power and shifted the centroid anterior in both alpha bands. Olanzapine shifted the centroid anterior in alpha 2 and beta 1 . Conclusions The increased microstate duration under perospirone and haloperidol was opposite to effects previously reported in schizophrenic patients, suggesting a key-lock mechanism. The opposite centroid changes induced by olanzapine and quetiapine compared to haloperidol might characterize the difference between conventional and atypical antipsychotics.

Keywords Pharmaco-EEG $\cdot$ Microstates $\cdot$ Schizophrenia . Key-lock principle $\cdot$ Chlorpromazine $\cdot$ Haloperidol . Olanzapine $\cdot$ Perospirone $\cdot$ Quetiapine $\cdot$ Risperidone

\section{Introduction}

Electroencephalography (EEG) offers high sensitivity to alterations of human functional brain states (Ozaki and Lehmann 2000). Specific EEG patterns related to various psychotropic drugs (Itil 1961; Fink 1968; Saito et al. 1983, 1993, 1998; Saletu 1987; Saletu et al. 1987; Galderisi et al. 
1996, see Mucci et al. (2006) for a review) and mental disorders (Itil et al. 1972; Saletu 1980; Saletu et al. 1986; Pascual-Marqui et al. 1999, see Hughes and John 1999 for a review) were previously reported. Saletu et al. (2002) investigated the relationship between alternations induced by mental disorders and by psychotropic drugs using an EEG source analysis called low-resolution brain electromagnetic tomography (LORETA; Pascual-Marqui et al. 1994, 1999). They found that some psychotropic drugs had EEG effects that seemed to counteract EEG changes that were seen in mental disorders, (i.e., schizophrenia and generalized anxiety disorder; this phenomenon has been termed 'key-lock principle'; Saletu-Zyhlarz et al. 2000; Saletu et al. 2002).

A compatibility or incompatibility with the key-lock hypothesis does not necessarily have implications for the efficiency of the drug but rather for the potential mechanisms of action. A key-lock pattern of drug response suggests that the drug acts upon processes that are present both in patients and controls, but in patients, these processes are quantitatively abnormal and thus dysfunctional. If a drug does have an antipsychotic effect but shows other than key-lock patterns of drug responses, it suggests that the drug affects processes that are qualitatively different or selectively present only in the responding patient group. The key-lock principle is thus not a conclusive approach to predict an antipsychotic action but is an incomplete but commonly accepted and testable general scheme to classify drug actions. However, as the clinical effects of the antipsychotic drugs often occur much later than the EEG drug effects and may involve mediating processes, the application of this scheme cannot be easily extended to the clinical symptoms.

In this paper, we investigated the EEG profile of six antipsychotic drugs: two conventional antipsychotic drugs and four new antipsychotic drugs, so-called 'atypical antipsychotic drugs'. Atypical antipsychotics are less specific for $\mathrm{D}_{2}$ receptors but also affect, among others, $5-\mathrm{HT}_{2 \mathrm{~A}}$ receptors and have fewer extrapyramidal side effects compared to conventional antipsychotic drugs (Stahl 2000). This study investigated how the effects of these drugs on the EEG of healthy controls compared to the EEG correlates of schizophrenia and whether conventional and atypical antipsychotic drugs had different EEG profiles.

EEG data represent a mixture of brain states, and psychotropic drugs might not affect all of them similarly. Therefore, these states need to be separated before drug effects can be studied effectively. Then, the drug effects may become apparent as alterations in energy, occurrence, or duration or as changes in the scalp distribution (EEG generators) of these EEG states. The most commonly used method to separate EEG states is by frequency transformation. The different frequency bands identified in EEG have been closely linked to many specific functional states such as sleep (Borbely and Achermann 1999) or development (John et al. 1980). An alternative and complementary approach is microstate analysis, where the time-domain EEG is parsed into sub-second periods of quasi-stable field topography (Lehmann et al. 1987). As in resting EEG, microstate topographies tend to concentrate around a small number of prototypical configurations; they can be efficiently clustered, and subsequent statistics can be done depending on the cluster membership of the microstates (Koenig et al. 1999, 2002; Strelets et al. 2003; Lehmann et al. 2005; Mueller et al. 2005).

To assess frequency domain drug effects, we used the root mean square (RMS) of spectral amplitude across electrodes as measure of global energy of the different frequency bands and the scalp location of the center of gravity of the power-maps to assess the spatial distribution. Microstate analysis was based on the duration, occurrence, and total time covered by the different microstate clusters and on global comparisons of the topography of the different microstate clusters between drug conditions.

We expected that in the measured quantitative EEG features, antipsychotic drugs had effects opposite to those seen in psychotic patients. Furthermore, we expected that some of these features would be different between typical and atypical antipsychotic drugs.

\section{Materials and methods}

\section{Ethical considerations}

This study was conducted in accordance with the Declaration of Helsinki (1964), and approved by the institutional ethical review board of Osaka Pharmacology Research Clinic, Osaka, Japan. All subjects participated voluntarily after being fully informed about the study and having given their written consents. Each subject was paid JPY 306,900 (equivalent to approximately 2700.00 USD) for the entire experiment.

\section{Subjects}

Fourteen right-handed, healthy male volunteers participated in this study. Subjects were recruited by the Osaka Clinical Research Organization for Medicaments (OCROM), where the subjects had voluntarily applied to participate in drug studies. The mean age was $24.1 \pm 4.1$ years (range 20 33 years). All subjects were in good health and showed normal findings in a general and neurological investigation. They did not have a drug allergy so far. Laboratory investigation (clinical chemistry, prolactin, blood cell count, virology for hepatitis $\mathrm{B}, \mathrm{C}$ and syphilis, and urinalysis) and electrocardiography showed normal findings. They had no 
brain organic abnormality according to a screening EEG. Apart from excluding cases with pathological EEGs, subjects were not selected based on EEG features. An interview performed by an experienced psychiatrist showed neither noticeable psychopathological symptoms nor a positive personal history for psychiatric disorders or substance abuse. No subjects had participated in drug studies within 4 months before the start of the study. They had not taken any drug within a week before entering the study and had not taken any drug that affects the central nervous system a month before entering the study. They entered the Osaka Pharmacology Research Clinic at 18:30 on the day before recording and stayed there until the morning after the recording day. They were only allowed to eat what was served at the clinic. Drinks containing alcohol, xanthine, and grapefruit as well as smoking were not allowed until all EEG recordings and physical examinations had been finished.

Thirteen subjects were smokers; average number of cigarettes smoked per day was 15.8 (SD, 4.5). These subjects might thus have experienced smoking withdrawal. However, because the design of the study (see below) counteracted effects of smoking abstention: (a) comparisons were made against a placebo baseline (assumingly producing the same abstention effects), (b) recordings were 2 to 3 weeks apart (preventing a habituation to the abstention), and (c) drug conditions (including placebo) were counterbalanced, eliminating possible sequential effects.

\section{Study design}

This study was designed as a single-blind, placebo-controlled study. All subjects went through seven EEG recording sessions separated each by a 2- or 3-week interval.

One of the following substances was applied orally in each session: chlorpromazine $(50 \mathrm{mg})$, haloperidol $(1 \mathrm{mg})$, olanzapine (1.25 mg), perospirone (4 mg; Kato et al. 1990; de Paulis 2002; Shiwa et al. 2003), quetiapine (33 mg), risperidone $(0.5 \mathrm{mg})$, and placebo. Chlorpromazine and haloperidol are typical antipsychotics, and olanzapine, perospirone, quetiapine, and risperidone are atypicals (Stahl 2000). Perospirone has been approved only in Japan. Its primary mode of action is through an antagonism of 5$\mathrm{HT}_{2 \mathrm{~A}}$ receptors and $\mathrm{D}_{2}$ receptors; besides, it acts as an agonist of 5-HT $1 \mathrm{~A}$ receptors (Kato et al. 1990; de Paulis 2002; Shiwa et al. 2003). Dosage was equivalent to of $1 \mathrm{mg}$ haloperidol, which, according to previous pharmaco-EEG studies of our laboratory, was enough to have an effect on the brain electrical activity while assuring subject's safety (Saito et al. 1983, 1993, 1998). Powders of test drugs were capsulated at the Kansai Medical University Hospital's Pharmacy. The controller of this study, Noriko Mochizuki, pharmacist of Osaka Pharmacology Research Clinic, confirmed undistinctiveness of the test drugs and ordered them in a crossover, Latin square design. Subjects took the test drug with $150 \mathrm{ml}$ of water at between 10:00 and 11:00, after a light meal.

\section{EEG recording}

Spontaneous resting EEG was recorded $2 \mathrm{~h}$ before the drug administration and at 2, 4, and $6 \mathrm{~h}$ after drug administration in each session. EEG was recorded from 19 scalp electrodes placed according to the International 10-20 system (Fp1, Fp2, F7, F3, Fz, F4, F8, T3, C3, Cz, C4, T4, T5, P3, Pz, P4, T6, O1, and $\mathrm{O} 2$ ) referred to linked earlobes. The EEG was amplified ( 0.3 to $60 \mathrm{~Hz}$ bandpass filter), digitized $(128 \mathrm{~Hz})$, and stored on hard disk using an EEG-1100 NIHON KODEN system (Nihon Koden, Tokyo, Japan). After each EEG recording, subjects were asked for the presence of side effects, which were then classified into slight, moderate, or severe sleepiness, fatigue, lassitude, and nausea. Furthermore, clinical chemistry, prolactin, and blood cell count were assessed.

\section{Quantitative EEG analysis}

From the stored EEG data, the first ten artifact-free 2-s epochs were selected. The selected data epochs were recomputed against average reference. For the following analyses, we used Brain Analyzer (version 1.05, Brain Products GmbH, Munich, Germany). EEG data recorded before drug administration was considered as habituation for recording of EEG and excluded from analysis.

\section{EEG microstate analysis}

For microstate analysis (Koenig et al. 1999, 2002), the data was digitally band-pass filtered from 2 to $20 \mathrm{~Hz}$. Global field power (GFP; Lehmann and Skrandies 1980), which quantifies the overall potential variance across the set of electrodes, was computed at each moment in time. As topography remains stable around peaks of GFP and changes during the troughs, only topographies at momentary maxima of the GFP were further analyzed (Lehmann et al. 1987; Strik and Lehmann 1993; Wackermann et al. 1993). As in previous works (Koenig et al. 1999, 2002; Strelets et al. 2003; Lehmann et al. 2005; Mueller et al. 2005), four optimally fitted microstate class topographies were obtained from all data of EEG using a modified version of the k-mean clustering algorithm (Pascual-Marqui et al. 1995). The algorithm was instructed to seek four classes of microstate topography and to assign each topography of EEG to one of these classes. The clustering criterion was global map dissimilarity (Lehmann and Skrandies 1980) between the original maps, disregarding map polarity. This number of classes has previously been found to be optimal using a cross validation criterion and 
was maintained to preserve comparability with earlier studies. Microstate class topographies were computed individually and averaged across subjects and time points using a permutation algorithm that maximized the common variance over subjects and time points (Koenig et al. 1999). Within each epoch, microstates were identified as continuous epochs where all topographies were assigned to the same class. Individual microstate profiles were computed for each class, consisting of mean microstate duration (duration), mean number of microstates per second (occurrence), and percentage of total analysis time occupied in that state (contribution). We calculated these values over all subjects and all time points after drug administration.

\section{EEG frequency domain analysis (EEG global spectral amplitudes and spatial centroids)}

All selected EEG epochs were frequency-transformed using the fast Fourier transformation. Spectral amplitudes were averaged across epochs for each electrode, subject, and condition. The RMS of spectral amplitude was computed across electrodes to obtain a measure of global amplitude for each frequency. The spatial configuration of each spectral amplitude map was quantified by computing the location of the center of gravity or 'centroids' (Wackermann et al. 1993) of the spectral amplitudes. Independently of total amplitude, these frequency domain map centroids reduce spectral distributions to two spatial parameters: the co-ordinate values of the location of the centroid on the anterior-posterior and on the left-right axes. As measuring units, electrode gridline distances (ED) were used, where one ED was the distance between two $10 \%$ lines of the modified 10-20 system (Fig. 1).

For each subject and condition, RMS and centroid locations were averaged within the following frequency bands: delta $(1.5-6.0 \mathrm{~Hz})$, theta $(6.5-8.0 \mathrm{~Hz})$, alpha $1(8.5-$ $10.0 \mathrm{~Hz})$, alpha $2(10.5-12.0 \mathrm{~Hz})$, beta $1(12.5-18.0 \mathrm{~Hz})$, beta $2(18.5-21.0 \mathrm{~Hz})$, and beta $3(21.5-30.0 \mathrm{~Hz})$. These frequency bands have previously been identified by factor analysis (Kubicki et al. 1979).

\section{Statistics}

\section{EEG microstate analysis}

Microstate configuration Separately for each of the four microstate classes, the configurations of their mean topographies over subjects and timepoints for each drug were compared between each antipsychotic drug and placebo using a randomization test (Manly 1991) based on the dissimilarity of topographies (Lehmann and Skrandies 1980). This randomization statistics used in this paper has been called topographic analysis of valiance and has

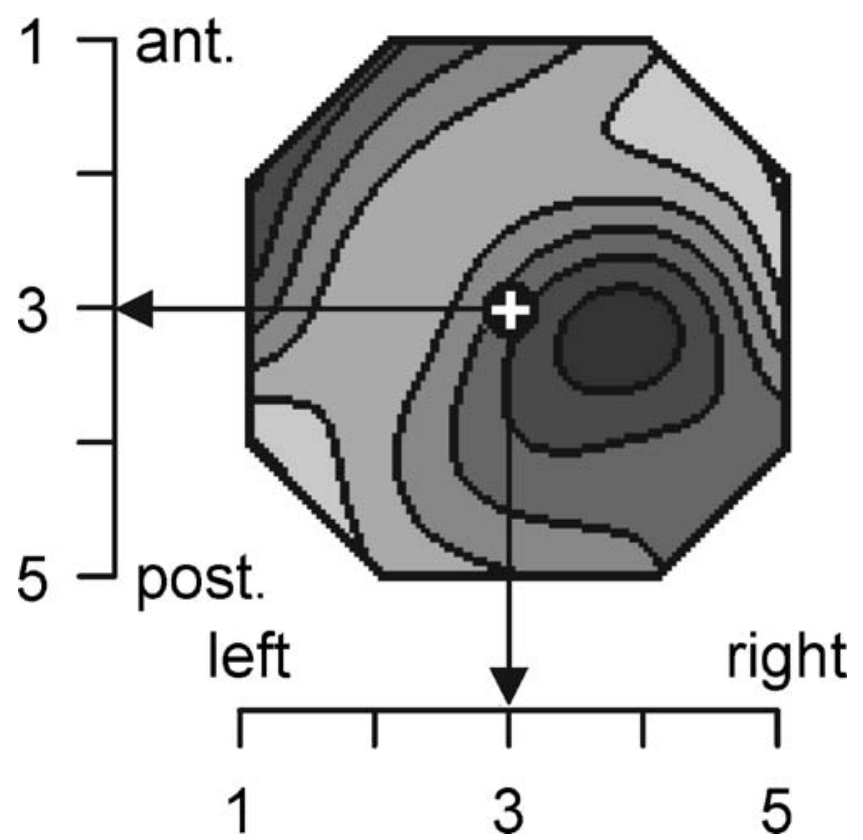

Fig. 1 Example for the quantification of the spatial distribution of spectral amplitude. Head seen from above, left ear left. Tick marks at the coordinate axes indicate electrode numbers, in the anterior-posterior direction (vertical axis) and in the left-right direction (horizontal axis). A sample distribution of spectral amplitudes is shown, darker values indicate more amplitude. The location of the centroid is indicated by the white cross. The configuration of the map is described by the anteriorposterior and left-right coordinates of the centroid

previously been extensively described and applied to EEG and event-related potential topographies (Strik et al. 1998). It estimates the probability that the observed difference between two mean topographies can be obtained by chance. (For a detailed description, see Strik et al. 1998.)

Microstate profiles For each microstate value (duration, occurrence, and contribution), repeated three-way analyses of variance (ANOVAs) were performed $(7 \mathrm{drugs} \times 3$ time points $\times 4$ microstate classes). When there were significant $(p<0.05)$ main effects or interactions including drug, we computed post hoc repeated three-way ANOVAs ( 2 drugs $\times$ 3 time points $\times 4$ microstate classes) comparing each antipsychotic drug separately with placebo. Significant drug effects in these post hoc ANOVAs were further explored using paired $t$ tests.

EEG frequency domain analysis (EEG global spectral amplitudes and spatial centroids) Repeated two-way ANOVAs ( 7 drugs $\times 3$ time points) were computed for RMS and for the anterior-posterior and left-right positions of the centroid locations in each frequency band. When significant $(p<0.05)$ or weak $(p<0.1)$ drug effects appeared, post hoc repeated two-way ANOVAs $(2$ drugs $\times 3$ time points) were performed comparing each antipsychotic drug separately to placebo. 


\section{Results}

All subjects went through all recording sessions without severe adverse drug reactions. Reported non-severe adverse drug reactions of antipsychotic drugs by subjects were slight sleepiness (during 39.3\% of all EEG recordings), moderate sleepiness $(9.5 \%)$, slight general fatigue $(2.4 \%)$, moderate general fatigue $(1.2 \%)$, slight lassitude of the upper limbs $(1.2 \%)$, moderate general lassitude $(1.2 \%)$, slight nausea $(1.2 \%)$, slight increase in plasma concentration of prolactin $(19.0 \%)$, and slight increase in white blood cells $(1.2 \%)$. We confirmed that all these adverse drug reactions disappeared in a reexamination.

Microstate analysis

\section{Microstate configuration}

Across the sample of 392 (14 subjects $\times 7$ drugs $\times 3$ time points) sets of EEG epochs, four microstate classes accounted on the average for $75.8 \%$ of the variance $(\mathrm{SD}=7.2 \%)$. Figure 2 shows the mean topographies of the four microstate classes obtained from all EEG data and the percent total time covered by each state. These overall results closely

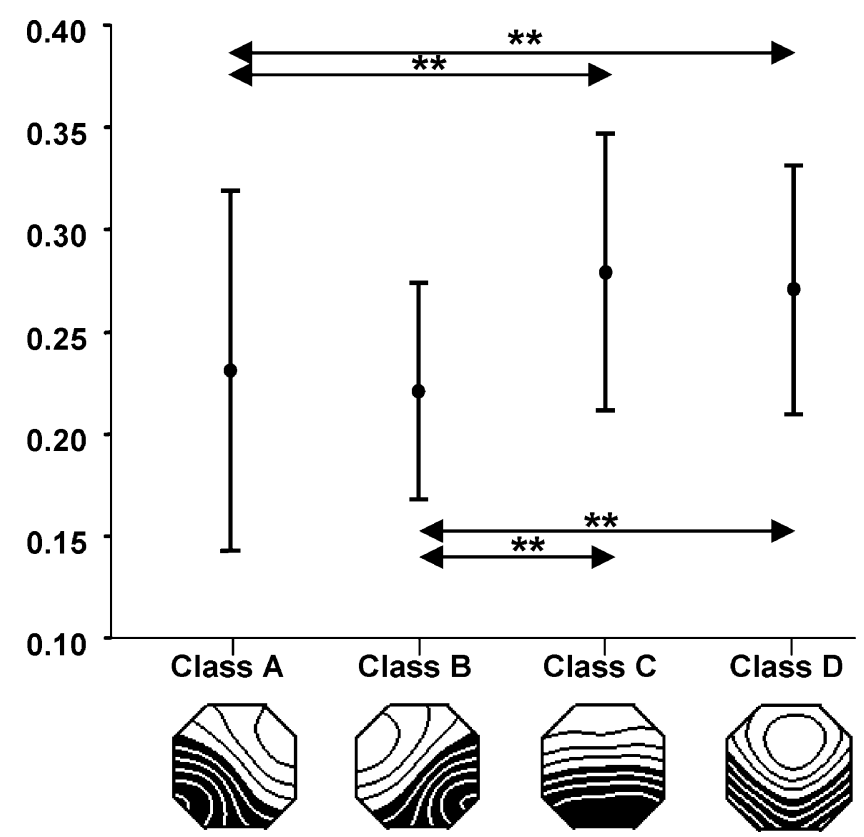

Fig. 2 Spatial configuration of four microstate classes (A-D) over all subjects and time points (maps in the lower part) and the contribution of each microstate class to the total analysis period (upper graph). The maps are shown as normalized isopotential maps, head seen from above, nose up. White and black map areas indicate opposite polarity against average reference. The dots in the upper graph indicate the contribution of each microstate class to the total analysis period (vertical axis) as function of microstate class (horizontal axis). The vertical bars are the $95 \%$ confidence interval. The horizontal arrows with two stars indicate significant $(p<0.05)$ differences of contribution between microstate classes replicated previous work (Koenig et al. 1999, 2002; Strelets et al. 2003; Lehmann et al. 2005; Mueller et al. 2005) and were therefore labeled and arranged in the same order as in previous publications (Koenig et al. 1999, 2002). Microstates of class A had a left occipital to right frontal orientation, class B was from right occipital to left frontal, class $\mathrm{C}$ had a symmetric occipital to prefrontal orientation, and class D was also symmetric but with a frontocentral and an occipital maximum of absolute voltage.

Figure 3 shows the configurations of four microstate classes (A-D) obtained from EEG data after each drug. Haloperidol, quetiapine, and risperidone significantly changed configurations of all four classes. Perospirone significantly changed configurations of three classes (A, C, and D). Chlorpromazine significantly changed configurations of two classes (C and D). Olanzapine significantly changed configurations of only class $\mathrm{C}$. Visual inspection indicated that many, but not all of these changes of microstate class topography, could be regarded as a clockwise rotation of the axis connecting the electrodes with the maximum and the minimum voltage.

\section{Microstate profiles}

Microstate duration Microstate duration varied between 64.42 and $77.50 \mathrm{~ms}$ for all drugs and four microstate classes. Repeated three-way ANOVAs ( 7 drugs $\times 3$ time points $\times 4$ microstate classes) showed a significant main effect of drug $(F=3.27 ; d f=6.78 ; p=0.0064)$. Repeated three-way ANOVAs between each antipsychotic drug and placebo yielded a significant main effect of drug between haloperidol and placebo $(F=6.99 ; d f=1.13 ; p=0.02)$, namely, the mean duration of all four microstate classes was significantly increased after haloperidol compared to placebo. Similarly, chlorpromazine had a tendency to increase the mean duration of all four microstate classes compared to placebo $(F=4.17$; $d f=1.13 ; p=0.0619)$. Figure 4 a shows the difference of mean duration of the four classes between each antipsychotic drug and placebo. There was a significant interaction between drug and microstate class $(F=3.16$; $d f=3.39 ; p=0.0354)$ when comparing perospirone and placebo. Post hoc paired $t$ tests identified a significant $(p=0.0314)$ increase in duration of class D after perospirone (76.01 ms) compared to placebo (70.77 ms; Fig. 4b).

Microstate occurrence Microstate occurrence varied between 3.20 and 3.94 counts/s for all drugs and four microstate classes. Repeated three-way ANOVAs $(7$ drugs $\times 3$ time points $\times 4$ microstate classes) showed a significant effect of drug $(F=3.07 ; d f=6.78 ; p=0.0095)$. Repeated three-way ANOVAs between each antipsychotic drug and placebo identified a significant main effect of drug between haloperidol and placebo $(F=5.93 ; d f=1.13 ; p=0.03)$, that 


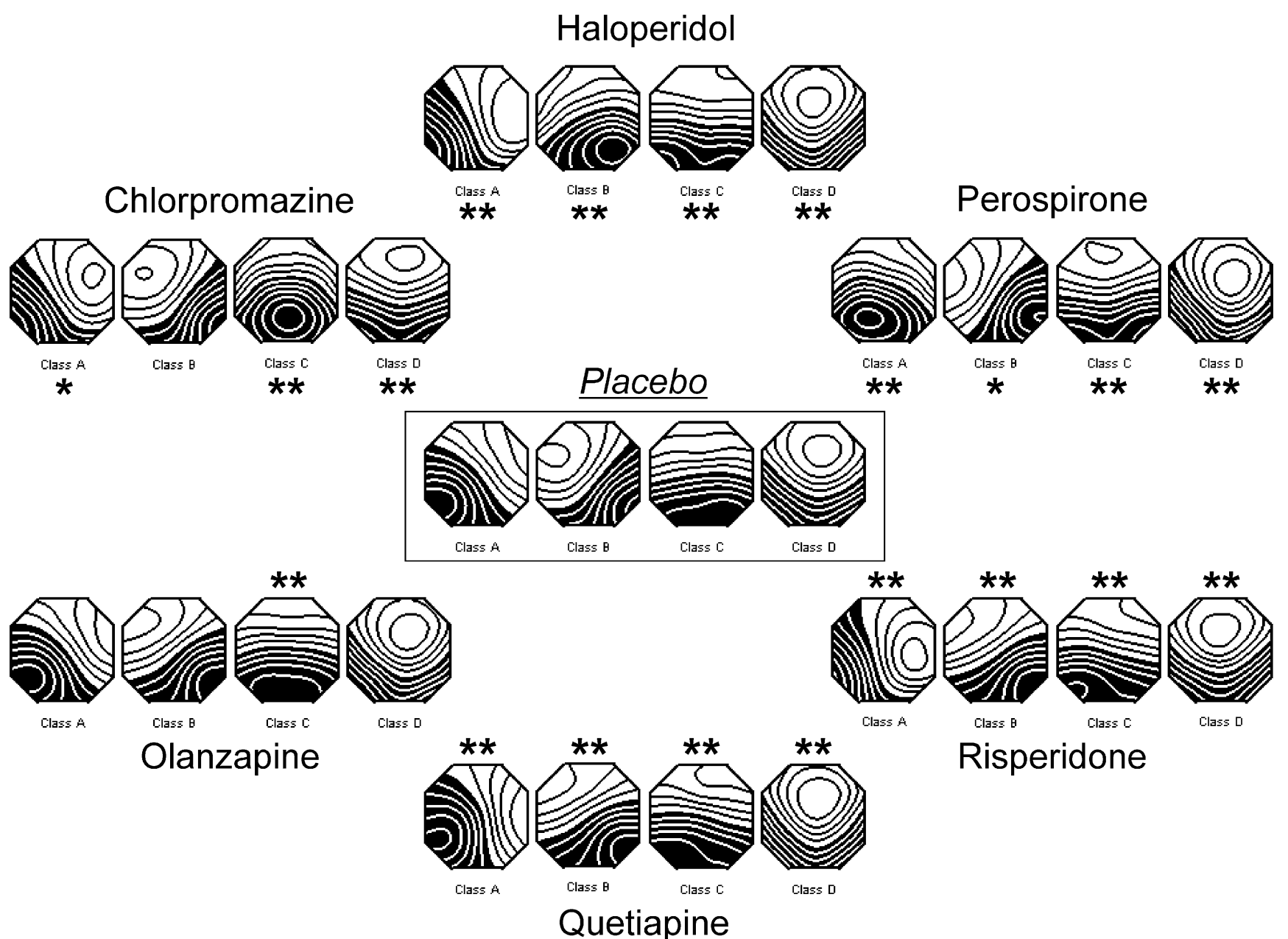

Fig. 3 Configurations of the four microstate classes (A-D) over all subjects and time points separately for each drug. Map display as in Fig. 2. Two stars indicate a significant $(p<0.05)$ difference between an

is, the mean occurrence of microstates was significantly decreased after haloperidol compared to placebo. Figure $4 \mathrm{c}$ shows the difference of mean occurrence of the four classes between each antipsychotic drug and placebo.

Microstate contribution Microstate contribution varied between 21.0 and $28.5 \%$ for all drugs and 4 microstate classes. Repeated three-way ANOVAs ( 7 drugs $\times 3$ time points $\times 4$ microstate classes) yielded a main effect of microstate class $(F=12.92 ; d f=3.39 ; p<0.0001$; Fig. 2$)$ and an interaction between microstate class, time, and drug. However, the post hoc repeated three-way ANOVAs comparing each drug to placebo failed to show significant effects including drug.

EEG frequency domain analysis

\section{EEG power analysis}

The overall ANOVAs on RMS yielded significant drug effects in the alpha 1 band $(F=4.3 ; d f=6.78 ; p=0.0009)$ and antipsychotic drug and placebo in a microstate class, one star indicates a tendency $(p<0.1)$

a weaker drug effect in the beta 1 band $(F=2.07 ; d f=6.78$; $p=0.0654)$. Post hoc tests indicated that haloperidol increased RMS in the alpha $1(F=5.66 ; d f=1.13 ; p=0.0334)$ and beta 1 $(F=6.28 ; d f=1.13 ; p=0.0263)$ band compared to placebo. Quetiapine significantly decreased RMS in the alpha 1 band compared to placebo $(F=6.22 ; d f=1.13 ; p=0.0269$; Fig. 5a).

\section{Gravity center of EEG}

The anterior-posterior centroid location showed significant drug effects in the alpha 2 band $(F=2.96 ; d f=6.78 ; p=$ $0.0118)$ and weak drug effects in alpha $1 \quad(F=1.92$; $d f=6.78 ; p=0.0879)$, beta $1(F=1.98 ; d f=6.78 ; p=0.078)$, and beta $2(F=2.09 ; d f=6.78 ; p=0.0635)$ bands. Post hoc tests indicated that quetiapine shifted the centroid anterior in both alpha bands (alpha 1, $F=751.42, d f=1.13$, $p<0.0001$; alpha 2, $F=16.32, d f=1.13, p=0.014$; Fig. 5b,c). A more anterior centroid location was also observed after olanzapine compared to placebo in alpha $2(F=22.4$; $d f=1.13 ; p=0.0003)$ and beta $1 \quad(F=4.68 ; d f=1.13$; $p=0.0498$; Fig. 5c,d). On the other hand, haloperidol, 

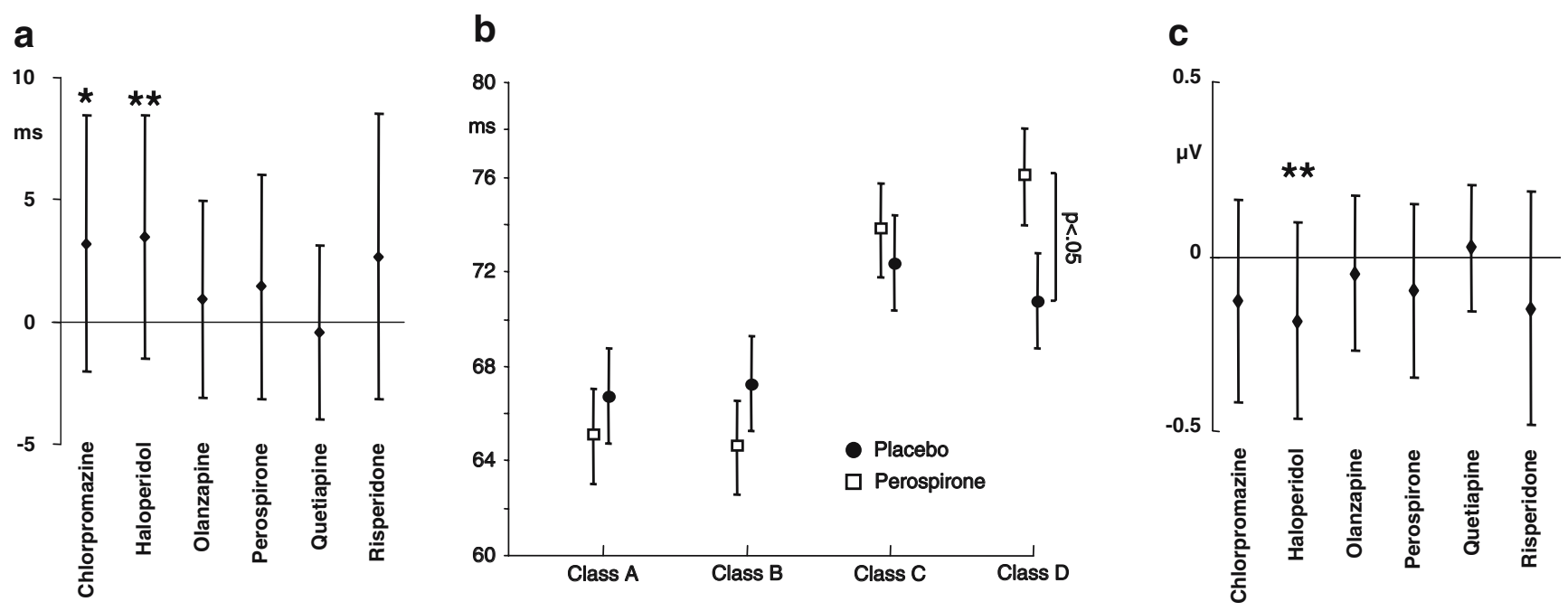

Fig. 4 Difference between each drug and placebo in mean microstate duration and occurrence. a Mean differences of microstate duration for the different drugs against placebo. Zero indicates the placebo level. The dots indicate mean values; the vertical bars indicate the standard deviation. Two stars indicate a significant $(p<0.05)$ difference between an antipsychotic drug and placebo in the mean microstate duration across classes; one star indicates a tendency $(p<0.1)$. b The mean microstate duration of perospirone (open squares) and placebo

compared to placebo, tended to shift the centroid posterior in the beta 1 band $(F=3.38 ; d f=1.13 ; p=0.0888$; Fig. $5 \mathrm{~d})$. No effects were observed on the left-right axis.

\section{Discussion}

We performed a study of pharmaco-EEG under two conventional antipsychotic drugs and four atypical antipsychotic drugs taken by healthy volunteers. The EEG was quantified by EEG microstate analysis, by total spectral amplitude, and by the location of the spatial gravity center of spectral amplitude. These methods yielded consistent differences between conventional and atypical antipsychotic drugs. Some of these effects were opposite to the abnormalities seen in schizophrenic patients and compatible the key-lock principle (Saletu-Zyhlarz et al. 2000; Saletu et al. 2002).

The analysis of EEG microstates was based on four classes of microstates constructed from all the measured EEG data. The contribution of each microstate class was similar to the previous studies that investigated mentally healthy subjects (Koenig et al. 2002).

All antipsychotic drugs we used in this study significantly affected the spatial configuration of some of the microstate classes. This means that the antipsychotic drugs changed the configuration of determined, transiently active neural networks. Particularly, all antipsychotic drugs affect- (filled circles) separately for each microstate class. Again, the vertical bars indicate the standard deviation. Perospirone significantly increased the duration of class D compared to placebo. $\mathbf{c}$ The differences between drugs and placebo of mean microstate occurrence per second across the four microstate classes can be seen. The dots indicate mean values; the vertical bars indicate the standard deviation. Significance indicated as in previous figures

ed class D. Microstate topographies have previously been shown to be abnormal in patients with schizophrenia selectively for class D (Koenig et al. 1999), suggesting that the drugs have a selective effect on brain states altered in schizophrenia.

Perospirone and haloperidol increased microstate duration compared to placebo. Perospirone selectively increased the duration of class D, while haloperidol increased the mean duration of all four microstate classes. In addition, chlorpromazine had a tendency to increase the mean duration of all four microstate classes compared to placebo. Previous studies on schizophrenic patients showed significant decrease in microstate duration of class B (Strelets et al. 2003; Lehmann et al. 2005) and D (Koenig et al. 1999; Lehmann et al. 2005) and a tendency toward decrease in mean microstate duration of all four microstate classes (Strelets et al. 2003; Lehmann et al. 2005) compared to control subjects. Lehmann et al. (2005) interpreted the decrease in microstate duration as a result of precocious termination of information processing in certain classes of mental operations.

Increased mean duration of all microstate classes was observed only after the conventional antipsychotic drugs haloperidol and chlorpromazine, which were both $\mathrm{D}_{2}$ receptor antagonists. The increase in mean microstate duration across microstate classes by conventional antipsychotic drugs may thus result from a blockade of $\mathrm{D}_{2}$ receptors. Longer duration of class $\mathrm{D}$ has been associated 


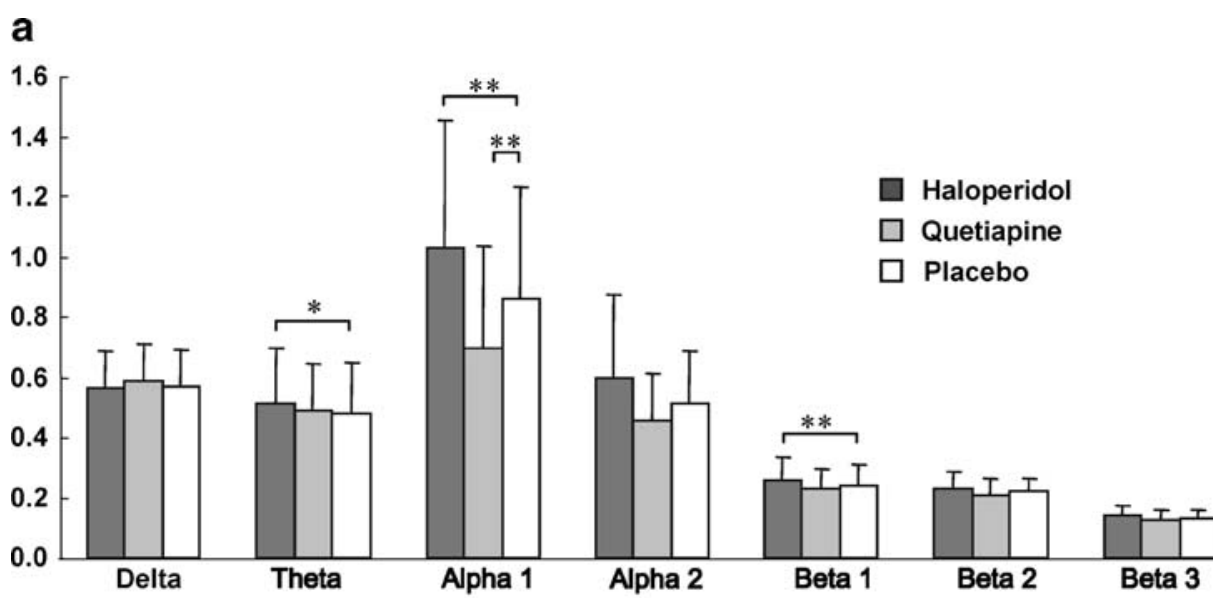

b

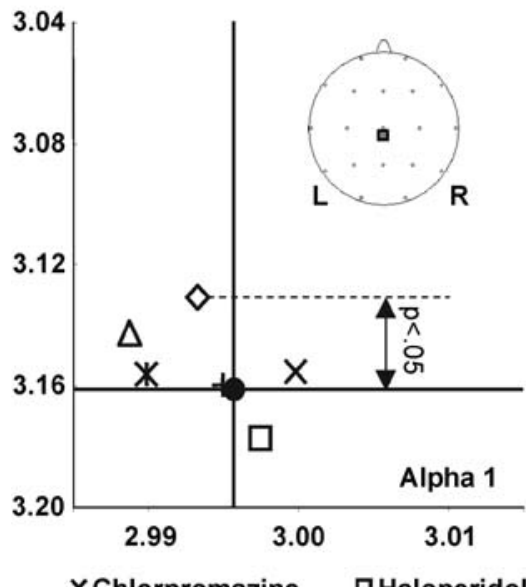

C

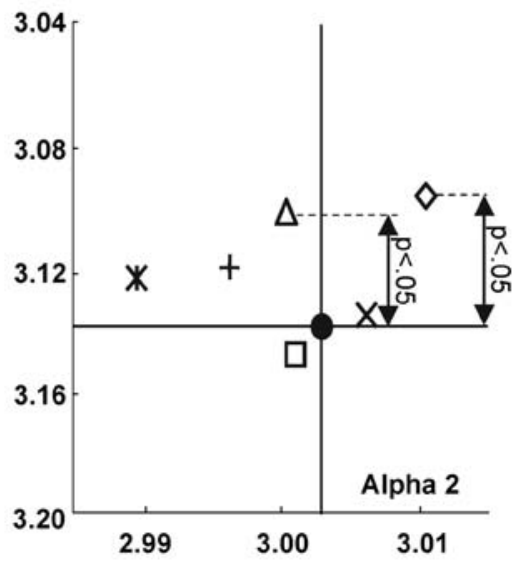

d

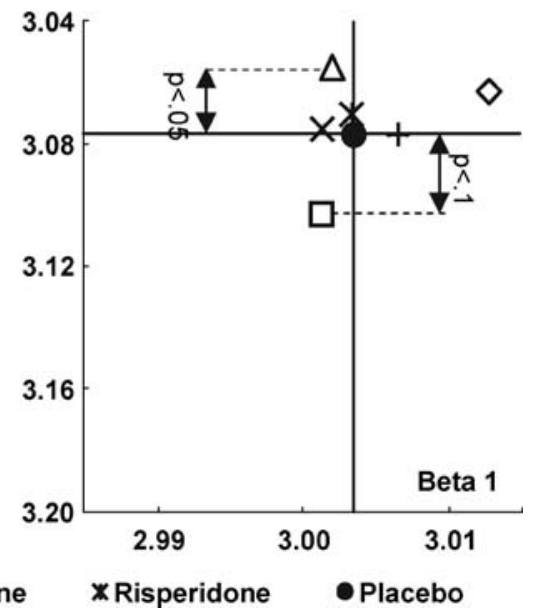

Fig. 5 Frequency domain drug effects. a The mean RMS of spectral amplitude of haloperidol, quetiapine, and placebo in seven frequency bands over all subjects and time points. The vertical bars indicate standard deviation. b-d The mean locations of spatial centroid of the spectral amplitude distribution of the different drugs and placebo in alpha 1 , alpha 2 , and beta 1 bands. The inset in $\mathbf{b}$ indicates the position, extent, and orientation of the area shown in $\mathbf{b}-\mathbf{d}$. The continuous lines indicate the centroid positions under placebo, the dotted lines and the arrows indicate drug-induced changes in centroid position with a significance level below $10 \%$ with less paranoid symptoms in schizophrenic patients (Koenig et al. 1999). Therefore, we can speculate (and eventually test) that the selective increase in duration of class D after perospirone has a particular effect against paranoid symptoms.

The employed drugs thus changed properties of transient brain electric states, and these changes depended consistently on the type of drug employed. Although the biological mechanism relating the drug's molecular actions to the microstate effects remains unknown, it became apparent that haloperidol, chlorpromazine, and perospirone acted differently upon EEG microstates from the other employed drugs. This diversity in microstate changes after intake of a neuroleptic drug in controls may thus correspond to the diversity observed in patients' responses to those drugs, which may be based on a diversity of biological mechanisms in schizophrenia, and these microstate changes may be compatible with the key-lock principle.

Itil (1961) proposed to classify psychotropic drugs by their profile of EEG spectral power changes. He classified antipsychotic drugs into a 'major neuroleptic type' and a 'major tranquilizer type'. The major neuroleptic type was characterized by an increase in slow and alpha waves. The major tranquilizer type was characterized by an increase in slow waves and a decrease in alpha and fast waves. Later, Saletu et al. (1987) classified antipsychotic drugs into 'nonsedative neuroleptics' and 'sedative neuroleptics' also based on spectral power analysis. Non-sedative neuroleptics were determined by increased alpha and alpha-adjacent beta 1 activity. Sedative neuroleptics were characterized by increased delta and theta activity, decreased alpha and, less consistently, increased concomitant fast beta activity. In this 
study, haloperidol increased RMS in alpha 1 and beta 1 band compared to placebo. This profile of haloperidol matched the characteristics of the major neuroleptic type and of the non-sedative neuroleptics. Slow alpha has been suggested to indicate a resting state (Isotani et al. 2001). Haloperidol may therefore enhance brain functions typically observed during awake resting. Quetiapine induced a decrease in alpha 1 power compared to placebo. This feature corresponds to Itil's major neuroleptic type and the Saletu's sedative neuroleptics, and it may suggest that quetiapine has a strong sedative effect.

Previous quantitative EEG comparing schizophrenic patients to healthy controls showed that patients had a decrease in activity in alpha 1 and an increase in activity and mean frequency in the beta band (Itil et al. 1972; Saletu 1980; Saletu et al. 1986). Our effects of haloperidol in healthy controls may be considered to be opposite to these results, because an increase in beta 1 (slow beta) power can be interpreted as decrease in mean frequency of the beta band. This phenomenon may thus be compatible with the key-lock principle.

Compared to placebo, quetiapine and olanzapine shifted the centroid anterior, quetiapine in both alpha bands and olanzapine in the alpha 2 and beta 1 band. The olanzapine results confirm a study by Hubl et al. (2001), who reported that olanzapine induced a frontal shift of electrical brain activity, significant in beta $2(16.0-19.5 \mathrm{~Hz})$ and a trend and in alpha $2(10.0-11.5 \mathrm{~Hz})$. The olanzapine effects in the beta 2 band were also similar to the results reported for clozapine, the prototype of an atypical antipsychotic drug (Galderisi et al. 1996). The EEG anteriorization induced by olanzapine and quetiapine may be related to effects of atypical antipsychotic drugs on frontal brain regions: atypical antipsychotics have a better effect on negative symptoms, which are considered as frontal lobe dysfunction. Furthermore, compared to the typical antipsychotics, they have an antagonistic action on $5-\mathrm{HT}_{2 \mathrm{~A}}$ receptors, which also affect frontal lobe functions.

Haloperidol was the only drug to shift alpha and beta band centroids posterior, although this was only a tendency in the beta 1 band (Fig. $4 \mathrm{~b}-\mathrm{d}$ ). The observation that haloperidol had effects on centroid location opposite to other antipsychotic drugs may be because haloperidol antagonizes $\mathrm{D}_{2}$ receptors more than atypical antipsychotics (Farde et al. 1992; Kasper et al. 2002). Dierks et al. (1995) reported a significant anteriorization of the EEG in schizophrenics compared to normals in beta 1 (12.0 $15.5 \mathrm{~Hz})$ and beta $2(16.0-19.5 \mathrm{~Hz})$ using an equivalent single dipole model (Lehmann and Michel 1989). This result is opposite to the anterior shift of haloperidol in this study. The strong antipsychotic effects of haloperidol may thus again be based on some key-lock mechanism, although the potential extrapyramidal side effects of haloperidol suggest some mismatch between the pathophysiological states of the patients and the counteracting drug effects.

Chlorpromazine and risperidone did not yield any significant result. This may be due to the low dosage of the test drugs or because these drugs specifically affect some abnormal brain functions usually not present in healthy controls. Compared to other studies, the dosage of the test drugs in this study was low, so it is difficult to compare these results with the results of other studies. For chlorpromazine and risperidone, it would thus be interesting to know whether in healthy controls, there is a dose-response relation and whether the dose used in this study was below the response threshold. In addition, it would be interesting to establish the EEG effects of different antipsychotic drugs on patients with schizophrenia and the relation of drug-related EEG changes with the clinical response.

Acknowledgment The authors would like to acknowledge the pharmacists of the Kansai Medical University Hospital's Pharmacy for the preparation of the test drugs and the colleagues of Department of Neuropsychiatry, Kansai Medical University for their help with the EEG recordings. This study complied with the current Japanese Pharmaceutical Affairs Laws. This study was supported by grants from the Mitsubishi Pharma Research Foundation.

\section{References}

Borbely AA, Achermann P (1999) Sleep homeostasis and models of sleep regulation. J Biol Rhythms 14:557-568

de Paulis T (2002) Perospirone (Sumitomo Pharmaceuticals). Curr Opin Investig Drugs 3:121-129

Dierks T, Strik WK, Maurer K (1995) Electrical brain activity in schizophrenia described by equivalent dipoles of FFT-data. Schizophr Res 14:145-154

Farde L, Nordstroem AL, Wiesel FA, Pauli S, Halldin C, Sedvall G (1992) Positron emission tomographic analysis of central $\mathrm{D}_{1}$ - and $\mathrm{D}_{2}$ - dopamine receptor occupancy in patients treated with classical neuroleptics and clozapine: relation to extrapyramidal side effects. Arch Gen Psychiatry 49:538-544

Fink M (1968) EEG classification of psychoactive compounds in man: review and therapy of behavioral associations. In: Efron DH, Cole JO (eds) Psychopharmacology: a review of progress 19571967. US Government Printing Office, Washington, pp 497-507

Galderisi S, Mucci A, Bucci P, Mignone ML, Maj M (1996) Multilead quantitative EEG profile of clozapine in resting and vigilancecontrolled conditions. Psychiatry Res 67:113-122

Hubl D, Kleinlogel H, Froelich L, Weinandi T, Maurer K, Holstein W, Czekalla J, Dierks T (2001) Multilead quantitative electroencephalogram profile and cognitive evoked potentials (P300) in healthy subjects after a single dose of olanzapine. Psychopharmacology 158:281-288

Hughes JR, John ER (1999) Conventional and quantitative electroencephalography in psychiatry. J Neuropsychiatry Clin Neurosci 11:190-208

Isotani T, Tanaka H, Lehmann D, Pascual-Marqui RD, Kochi K, Saito N, Yagyu T, Kinoshita T, Sasada K (2001) Source localization of EEG activity during hypnotically induced anxiety and relaxation. Int J Psychophysiol 41:143-153 
Itil TM (1961) Electroencephalographische Befunde zur Klassifikation neuro- und thymoleptischer Medikamente. Med Exp 5:347-363

Itil TM, Saletu B, Davis S (1972) EEG findings in chronic schizophrenics based on digital computer period analysis and analog power spectra. Biol Psychiatry 5:1-13

John ER, Ahn H, Prichep LS, Trepetin M, Brown D, Kaye H (1980) Developmental equations for the electroencephalogram. Science 210:1255-1258

Kasper S, Tauscher J, Willeit M, Stamenkovic M, Neumeister A, Kuefferle B, Barnas C, Stastny J, Praschak-Rieder N, Pezawas L, de Zwaan M, Quiner S, Pirker W, Asenbaum S, Podreka I, Bruecke T (2002) Receptor and transporter imaging studies in schizophrenia, depression, bulimia and Tourette's disorder: implications for psychopharmacology. World J Biol Psychiatry 3:133-146

Kato T, Hirose A, Ohno Y, Shimuzu H, Tanaka H, Nakamura M (1990) Binding profile of SM-9018, a novel antipsychotic candidate. Jpn J Pharmacol 54:478-481

Koenig T, Lehmann D, Merlo MCG, Kochi K, Hell D, Koukkou M (1999) A deviant EEG brain microstate in acute, neurolepticnaïve schizophrenics at rest. Eur Arch Psychiatry Clin Neurosci 249:205-211

Koenig T, Prichep L, Lehmann D, Sosa PV, Braeker E, Kleinlogel H, Isenhart R, John ER (2002) Millisecond and millisecond, year by year: normative EEG microstates and developmental stages. NeuroImage 16:41-48

Kubicki S, Herrmann WM, Fichte K, Freund G (1979) Reflections on the topics: EEG frequency bands and regulation of vigilance. Pharmakopsychiatr Neuropsychopharmakol 12:237-245

Lehmann D, Michel CM (1989) Intracerebral dipole sources of EEG FFT power maps. Brain Topogr Fall-Winter 2:155-164 (erratum in: Brain Topogr 1990 Summer 2:311)

Lehmann D and Skrandies W (1980) Reference-free identification of components of checkerboard-evoked multichannel potential fields. Electroencephalogr Clin Neurophysiol 48:609-621

Lehmann D, Ozaki H, Pal I (1987) EEG alpha map series: brain micro-states by space-oriented adaptive segmentation. Electroencephalogr Clin Neurophysiol 67:271-288

Lehmann D, Faber PL, Galderisi S, Hermann WM, Kinoshita T, Koukkou M, Mucci A, Pascual-Marqui RD, Saito N, Wackermann J, Winterer G, Koenig T (2005) EEG microstate duration and syntax in acute, medication-naïve, first-episode schizophrenia: a multi-center study. Psychiatry Res 138:141-156

Manly BFJ (1991) Randomization and Monte Carlo methods in biology. Chapman and Hall, London

Mucci A, Volpe U, Merlotti E, Bucci P, Galderisi S (2006) PharmacoEEG in psychiatry. Clin EEG Neurosci 37:81-98

Mueller TJ, Koenig T, Wackermann J, Kalus P, Fallgatter A, Strik WK, Lehmann D (2005) Subsecond changes of global brain state in illusory multistable motion perception. J Neural Transm 112:565-576

Ozaki H, Lehmann D (2000) EEG reconsidered: from neuroelectric signals to human conscious experience. Jpn J Clin Neurophysiol 28:15-17

Pascual-Marqui RD, Michel CM, Lehmann D (1994) Low resolution electromagnetic tomography: a new method for localizing electrical activity in the brain. Int J Psychophysiol 18:49-65

Pascual-Marqui RD, Michel CM, Lehmann D (1995) Segmentation of brain electrical activity into microstates: model estimation and validation. IEEE Trans Biomed Eng 42:658-665
Pascual-Marqui RD, Lehmann D, Koenig T (1999) Low resolution brain electromagnetic tomography (LORETA) functional imaging in acute, neuroleptic-naive, first-episode, productive schizophrenia. Psychiatry Res 30:169-179

Saito M, Kagono Y, Suitsu N, Yamamoto Y, Kinoshita T, Ohya D, Fukui Y, Hashimoto C (1983) A study on anti-psychotic drugs by the aid of quantitative EEG and multivariate analysis-doses and action trait of haloperidol. Jpn J Neuropsychopharmacol 5:843-856

Saito N, Okajima Y, Isotani T, Yagyu T, Nobuhara K, Nishimura T, Fukushima M, Kuginuki T, Kinoshita T, Saito M (1993) Early prediction and orientation of the clinical effects of a new antipsychotic compound, risperidone, in human: a quantitative pharmaco-EEG study. Jpn J Neuropsychopharmacol 15:693-703

Saito A, Kuginuki T, Fukushima M, Saito M (1998) Pharmaco-EEG study of perospirone, a D2 and 5-HT2 antagonist, in healthy male volunteers. Jpn J EEG EMG 26:205-215

Saletu B (1980) Central measures in schizophrenia. In: Van Praag HM, Lader MH, Rafaelsen OJ, Sachar EJ (eds) Handbook of biological psychiatry part II. Brain mechanisms and abnormal behaviorpsychophysiology. Marcel Dekker, New York, pp 97-144

Saletu B (1987) The use of pharmaco-EEG in drug profiling. In: Hindmarch I, Stonier PD (eds) Human psychopharmacology: measures and methods, vol 1. Wiley, Chichester, pp 172-200

Saletu B, Kuefferle B, Gruenberger J, Anderer P (1986) Quantitative EEG, SPEM, and psychometric studies in schizophrenics before and during differential neuroleptic therapy. Pharmacopsychiatry 19:434-437

Saletu B, Anderer P, Kinsperger K, Gruenberger J (1987) Topographic brain mapping of EEG in neuropsychopharmacology: part II. Clinical applications (pharmaco EEG imaging). Methods Find Exp Clin Pharmacol 9:385-408

Saletu B, Anderer P, Saletu-Zyhlarz GM, Pascual-Marqui RD (2002) EEG topography and tomography in diagnosis and treatment of mental disorders: evidence for a key-lock principle. Methods Find Exp Clin Pharmacol 24(Suppl D):97-106

Saletu-Zyhlarz GM, Anderer P, Berger P, Gruber G, Oberndorfer S, Saletu B (2000) Nonorganic insomnia in panic disorder: comparative sleep laboratory studies with normal controls and placebo-controlled trials with alprazolam. Hum Psychopharmacol $15: 241-254$

Shiwa T, Amano T, Matsubayashi H, Seki T, Sasa M, Sakai N (2003) Perospirone, a novel antipsychotic agent, hyperpolarizes rat dorsal raphe neurons via 5-HT1A receptor. J Pharmacol Sci 93:114-117

Stahl SM (2000) Antypsychotic agents. In: Essential psychopharmacology: neuroscientific basis and practical applications, 2nd edn. Cambridge University Press, Cambridge, pp 401-458

Strelets V, Faber PL, Golikova J, Novototsky-Vlasov V, Koenig T, Gianotti LRR, Gruzelier JH, Lehmann D (2003) Chronic schizophrenics with positive symptomatology have shortened EEG microstate durations. Clin Neurophysiol 114:2043-2051

Strik WK, Lehmann D (1993) Data-determined window size and space-oriented segmentation of spontaneous EEG map series. Electroencephalogr Clin Neurophysiol 87:169-174

Strik WK, Fallgatter AJ, Brandeis D, Pascual-Marqui RD (1998) Three-dimensional tomography of event-related potentials during response inhibition: evidence for phasic frontal lobe activation. Electroencephalogr Clin Neurophysiol 108:406-413

Wackermann J, Lehmann D, Michel CM, Strik WK (1993) Adaptive segmentation of spontaneous EEG map series into spatially defined microstates. Int J Psychophysiol 14:269-283 